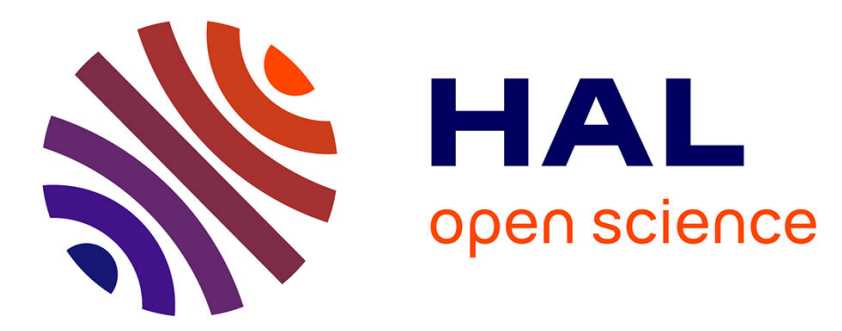

\title{
Observation des pratiques audiovisuelles avec de jeunes enfants en crèche
}

\author{
Christian Hudelot, Astrid van Der Straten, Chantal Simon-Caracci
}

\section{To cite this version:}

Christian Hudelot, Astrid van Der Straten, Chantal Simon-Caracci. Observation des pratiques audiovisuelles avec de jeunes enfants en crèche. L'enfant dans le lien social: Perspectives de la psychologie du développement, èrès, pp.181-187, 2003. halshs-00103644

\section{HAL Id: halshs-00103644 \\ https://shs.hal.science/halshs-00103644}

Submitted on 4 Oct 2006

HAL is a multi-disciplinary open access archive for the deposit and dissemination of scientific research documents, whether they are published or not. The documents may come from teaching and research institutions in France or abroad, or from public or private research centers.
L'archive ouverte pluridisciplinaire HAL, est destinée au dépôt et à la diffusion de documents scientifiques de niveau recherche, publiés ou non, émanant des établissements d'enseignement et de recherche français ou étrangers, des laboratoires publics ou privés. 


\section{Observations des pratiques audiovisuelles avec de jeunes enfants en crèche}

Christian Hudelot*, Astrid van der STRATEN*, Chantal SimOn-CARACCI*

\section{Une recherche commandée par le conseil général de Seine-Saint-Denis}

L'utilisation de l'audiovisuel et l'accroissement du nombre des très jeunes spectateurs amènent les responsables de la santé et de l'éducation à s'interroger sur les effets de ces pratiques sur la santé de l'enfant, son développement psychologique, affectif et social.

Une précédente étude qui portait sur les pratiques audiovisuelles dans les crèches et l'impact de l'image sur les jeunes enfants ${ }^{1}$, avait abouti, entre autres, au constat d'une utilisation professionnelle non négligeable du magnétoscope et de la vidéo dans les crèches de Seine-SaintDenis $(33 \%)$, mais inférieure à celle de la radiocassette $(77 \%)$, de l'appareil photo $(73 \%)$ et du projecteur de diapositives $(66 \%)$.

L'étude montrait également que dans leur grande majorité, les professionnels estimaient avoir un rôle à jouer dans l'éducation à l'image et que ce rôle n'était pas réservé aux seuls parents ou à l'école maternelle. Toutefois ces professionnels reconnaissaient un besoin de formation, similaire à ce qui se fait dans le Département pour l'accès au livre.

Enfin, quand il leur fut demandé de choisir parmi les activités celles qui permettent, dans le développement des échanges (avec les autres enfants ou avec l'adulte), de favoriser l'expression verbale, de développer les capacités d'attention ou de développer l'imaginaire de l'enfant les professionnels ne classèrent la vidéo qu'en troisième position après le livre et la projection de diapositives. Ce jugement reposait en partie sur l'attitude manifestée par les enfants à l'occasion de ces diverses activités, mais l'appréciation restait impressionniste. Il fut donc décidé d'objectiver davantage les observations en centrant l'étude sur la réception de productions narratives.

\section{Objectifs}

Il s'agit d'évaluer “l'impact" des médias audiovisuels dans l'accès à la culture narrative et de proposer, à partir de l'étude, une réflexion sur le jeune enfant et l'image, afin de sensibiliser le

\footnotetext{
* Groupe COLA (Corps et langage) du LEAPLE -UMR 8606, C.N.R.S. \& Université René Descartes Paris 5.

${ }^{1}$ Réalisée et publiée en mars 1999 par Denise Josse et Pascale Mirailles.
} 
personnel des crèches à ce thème et de l'aider dans une utilisation lucide des moyens audiovisuels.

On s'interrogera plus largement sur le rôle des nouveaux médias dans l'accès du très jeune enfant à la culture. Il s'agit ni de rejeter les nouveaux moyens d'expression, ni non plus de les banaliser mais de comprendre comment ils s'articulent à d'autres moyens d'accès à la culture - et en particulier le livre - et comment les adultes peuvent servir de médiateur entre les enfants et eux.

\section{Dispositif}

Il s'agit de comparer les modes d'entrée dans la culture narrative à partir de trois types de support de la même histoire (Max et les Maximonstres de Maurice Sendak) et de deux types d'attitude, neutre ou incitative du lecteur du livre ou du présentateur de la projection (assuré par un membre de l'équipe).

\begin{tabular}{|c|c|c|}
\hline types de support & Adulte incitatif & Adulte présentateur \\
\hline livre & & \\
\hline vidéo & & \\
\hline diapo & & \\
\hline
\end{tabular}

Après chaque présentation, une adulte familière des enfants, absente lors de la première phase, vient solliciter chaque groupe sur le récit qui vient d'être montré. Une analyse classique de restitution-reformulation de récit permettra d'évaluer pour partie l'impact immédiat des différents supports.

Dans chaque crèche visitée (12 sont prévues au total), les enfants de la section des grands (2;6 à $3 ; 0)$ sont réunis par groupe de quatre et sont filmés dans l'une des trois situations. On enregistre d'une part la phase d'écoute, et d'autre part la phase de restitution à un adulte familier.

\section{Analyse}

L'objectif est d'évaluer d'une part l'intérêt et l'attention de l'enfant à l'écoute et d'autre part son comportement et sa capacité à raconter l'histoire à la restitution.

La méthode consiste à élaborer deux grilles d'analyse rendant compte des conduites mimoposturo-gestuelles et verbales une grille lors de l'écoute ou du visionnement de l'histoire, et à l'occasion de sa restitution. 
Elaboration des catégories de la grille:

A partir d'un premier échantillon des enregistrements par caméra vidéo de 24 enfants, on a procédé au relevé de tous les comportements des enfants et on a opéré des regroupements de ces comportements en une série de grandes attitudes.

Une grille d'analyse de la composante proprement verbale tant de l'écoute que de la restitution est également en en cours d'élaboration mais ne sera pas présentée ici.

\section{Principe de la grille d'analyse}

Un tableau permet de noter les conduites de chaque enfant en fonction du déroulement de la séance. La première colonne note le temps d'après l'horloge de la bande par tranches de 10 secondes (durée adaptée aux modifications des attitudes définies ci-dessus) puis on trouve une colonne par enfant. Les lignes présentent dans la succession des unités de temps les différentes attitudes manifestées par chacun des enfants. Une grille est étable pour chaque groupe de quatre enfants.

Codage

Soit les attitudes suivantes et leurs codages :

\section{A l'ecoute :}

ATT $=$ attentif : regarde le support ou l'adulte

$=$ reste calme et immobile, le regard orienté vers le livre, l'écran ou le lecteur.

Pour être noté attentif il faut au moins 6" d'attention, consécutives ou non, dans la tranche de temps considérée.

PRT = participatif : participe par le geste ou la voix

$=$ présente un comportement de participation à la tâche dans la tranche de temps considérée

ex: montre du doigt, s'approche du livre, tourne les pages, sourit, rit, bat le rythme en même temps que la musique, mime, dit quelquechose (dénomme, commente...ex : «bateau! », « pas beau! »)

$\mathbf{E M B}=$ embarrassé pour répondre ou raconter 
= n'arrive pas à répondre à la question posée par l'adulte : se tortille, sourit, se tripote les mains, hausse les épaules,

INA = inattentif à la tâche mais reste calme

= est calme et immobile mais regarde ailleurs que le support ou l'adulte ou l'enfant qui raconte, ex: regarde un autre enfant, ses baskets, son doudou, par la fenêtre, tripote sa tétine, ses lacets, son pull,

Pour être noté inattentif il faut au moins 6" d'inattention, consécutives ou non, dans la tranche de temps considérée.

DISP $=$ dissipé : inattentif + turbulent

$=$ est inattentif à la tâche mais en plus est aussi agité, ex. : se lève, se rassied, s'allonge, se frappe les jambes, s'étire, marche, court.

QUT $=$ quitte volontairement le groupe

$=$ se lève et s'en va plus loin dans la pièce et par ce fait sort du champ de la caméra.

HOP $=$ hors propos : parle d'autre chose, ou montre autre chose

$=$ parle d'autre chose que de la tâche, ex. « la totote à Léa »; montre autre chose qu'un objet de la tâche, ex. quelqu'un qui passe dans le couloir, une affiche accrochée au mur, un jouet dans la pièce.

$\mathbf{N C}=$ non codable parce que non visible à l'écran

= est dans le groupe et dans la tâche mais devient peu ou pas visible à l'écran parce qu'un autre enfant s'est placé devant lui.

Ce cas de figure est noté au dépouillement mais déduit lors du calcul des pourcentages.

\section{A la restitution :}

On retrouve toutes les attitudes précédentes auxquelles on ajoute deux attitudes spécifiques :

$\mathbf{R A V}=$ parle ou $«$ raconte $»$ l'histoire lue ou vue en vidéo

= parle du contenu de l'histoire (personnage, événement, etc.. «c'est les monstres », «y dansent »)

mais aussi : du support («la cassette! »), des individus présents («le monsieur, il a raconté »), de la gestion de la possession du livre ou des tours de parole (« c'est à moi! »); invente ou dit n'importe quoi (ex: « y avait un oeuf, bleu! ») 
dit « je sais pas »

$\mathbf{R A G}=$ s'exprime ou $\ll$ raconte $»$ par des gestes

$=$ ne parle pas mais fait des gestes évoquant ou désignant un élément de l'histoire

ex.: montre du doigt le téléviseur, touche du doigt ou tapote une image du livre puis regarde l'adulte, mime ce que font les monstres (sauter, danser, grogner), répond à une question de l'adulte en faisant oui ou non de la tête.

Exemples 1. Comportements à l'écoute du livre Groupe 1 (Crèche départementale de Drancy) lecteur incitatif.

\begin{tabular}{|c|c|c|c|c|}
\hline temps & SERENA & LEA & FAÏM & YANIS \\
\hline 10 & ATT & ATT & ATT & ATT \\
\hline 20 & ATT & ATT & ATT & ATT \\
\hline 30 & ATT & PRT & PRT & ATT \\
\hline 40 & ATT & PRT & PRT & ATT \\
\hline 50 & ATT & PRT & PRT & PRT \\
\hline 01.00 & ATT & PRT & PRT & PRT \\
\hline 10 & INA & PRT & PRT & ATT \\
\hline 20 & ATT & ATT & ATT & ATT \\
\hline 30 & ATT & PRT & ATT & ATT \\
\hline 40 & INA & ATT & ATT & ATT \\
\hline 50 & INA & ATT & ATT & ATT \\
\hline 02.00 & INA & PRT & ATT & ATT \\
\hline 10 & INA & PRT & PRT & ATT \\
\hline 20 & ATT & ATT & INA & ATT \\
\hline 30 & ATT & ATT & ATT & ATT \\
\hline 40 & ATT & ATT & ATT & ATT \\
\hline 50 & ATT & ATT & INA & ATT \\
\hline 03.00 & ATT & INA & INA & ATT \\
\hline
\end{tabular}

Exemples 2. Comportements à la restitution Groupe 1 (Crèche Départementale de Drancy)

\begin{tabular}{|c|c|c|c|c|}
\hline temps & & & & \\
\hline 08.00 & ATT & RAG & ATT & ATT \\
\hline 10 & ATT & RAG & ATT & PRT \\
\hline 20 & NC & RAG & INA & PRT \\
\hline 30 & NC & RAG & INA & ATT \\
\hline 40 & NC & INA & INA & ATT \\
\hline 50 & INA & INA & NC & ATT \\
\hline 09.00 & RAV & PRT & INA & PRT \\
\hline 10 & RAV & PRT & ATT & ATT \\
\hline
\end{tabular}




\begin{tabular}{|c|c|c|c|c|}
\hline 20 & RAV & PRT & ATT & ATT \\
\hline 30 & RAV & PRT & PRT & NC \\
\hline 40 & RAV & PRT & ATT & ATT \\
\hline 50 & RAV & PRT & INA & PRT \\
\hline 10.00 & NC & RAG & ATT & PRT \\
\hline 10 & RAV & PRT & PRT & PRT \\
\hline 20 & ATT & PRT & ATT & RAG \\
\hline 30 & ATT & ATT & INA & RAG \\
\hline 40 & RAV & ATT & INA & RAG \\
\hline 50 & ATT & ATT & INA & RAG \\
\hline
\end{tabular}

\section{Quelques résultats:}

Les premiers résultats qui concernent six groupes d'enfants dans deux crèches différentes portent uniquement sur deux supports, le livre et la vidéo. Ils montrent de grandes différences entre les deux média.

\begin{tabular}{|c|c|}
\hline À l'écoute & Lors de la restitution \\
\hline $\begin{array}{l}\text { Pendant la lecture du livre, les enfants sont } \\
\text { très attentifs, très calmes, souvent participatifs. } \\
\text { Ils sont tournés vers l'adulte plutôt que vers } \\
\text { leurs camarades } \\
\text { Pendant la projection vidéo, ils présentent } \\
\text { une grande diversification de comportements: } \\
\text { tantôt attentifs et calmes, souvent distraits ou } \\
\text { turbulents, parfois participatifs et même } \\
\text { somnolents. }\end{array}$ & $\begin{array}{l}\text { Après la lecture du livre, les enfants sont } \\
\text { tous pressés de raconter et avides de tenir le } \\
\text { livre ou en cherchent un autre si on le leur a } \\
\text { retiré. Ils racontent spontanément soit à } \\
\text { l'adulte, soit aux autres enfants. } \\
\text { Après la projection vidéo, ils racontent } \\
\text { difficilement quelle qu'ai été leur attitude lors } \\
\text { de la projection. Ils ne racontent qu'à l'adulte, } \\
\text { et uniquement lorsqu'ils sont sollicités. }\end{array}$ \\
\hline
\end{tabular}

Le travail se poursuit actuellement dans une double direction. Sur le plan de la collecte des données, la réalisation d'un jeu de diapositives à partir de du livre à permis de procéder aux premiers enregistrements de séances de diapositives avec quatre groupes d'enfants. Nous avons été frappés, au cours de ces séances, surtout pendant la projection où l'adulte adopte une posture incitative, de voir combien les enfants étaient prompts à faire des commentaires sur l'image projetée, sans que cela ne préjuge nécessairement une meilleure « restitution ».

Par ailleurs, la transcription in extenso de la phase de restitution est actuellement en cours et devrait permettre d'abord d'affiner, sur le plan proprement verbal les grilles précédentes, mais 
aussi, et surtout de voir dans quelle mesure le mode de support (livre, cassette ou vidéo) ou de présentation (neutre ou incitative) influent sur les performances « narratives » des jeunes enfants. 